\title{
Le traitement de la leucémie myéloïde chronique par les interférons $\alpha$
}

Alors que le pronostic de la leucémie myéloïde chronique n'a pas changé depuis trente ans, l'interféron $\alpha$ constitue un réel espoir thérapeutique. Seul ou en combinaison avec une chimiothérapie, il entraîne une rémission complète sur le plan hématologique dans 60 à $80 \%$ des cas, associée à une disparition du marqueur cytogénétique de la maladie (le chromosome Philadelphie) dans 15 à $35 \%$ des cas. L'amplification par PCR de l'ADN hybride transcrit du gène réarrangé bcr-abl reste cependant généralement positive, même en cas de rémission cytogénétique. Les, effets du traitement par l'interféron $\alpha$ sur la survie des malades ne peuvent encore être évalués avec certitude, mais semblent favorables, surtout dans les cas d'obtention d'une rémission cytogénétique. Ce nouveau traitement mérite donc d'être proposé aux malades qui ne peuvent bénéficier de greffe de moelle allogénique, seul traitement potentiellement curateur actuellement à la disposition des médecins.

\section{François Guilhot}

\section{ADRESSE}

F. Guilhot : professeur d'hématologie. Service d'hématologie et d'oncologie médicale, hôpital Jean-Bernard, La Miletrie, 86021 Poitiers, France. a leucémie myéloïde chronique (LMC) est une hémopathie maligne chronique, appartenant au groupe des syndromes myéloprolifératifs et consécutive à l'atteinte d'un progéniteur pluripotent. Il s'agit d'un processus monoclonal caractérisé par une anomalie acquise du caryotype : le chromosome Philadelphie ou Ph. Il correspond à une translocation entre le chromosome 22 dont le grand bras est raccourci et le chromosome 9. Les techniques chromosomiques ont permis de préciser la localisation des points de cassure sur les chromosomes 9 et 22, respective- ment en q34 et q11. Cette translocation réciproque, $\mathrm{t}(9 ; 22)$ ( $\mathrm{q} 34$; q11), aboutit à la mise en continuité de la partie centromérique 5' de la région bor du chromosome 22 avec la partie C-terminale $3^{\prime}$ du protooncogène $a b l$ situé sur le 9 . Le gène hybride ainsi constitué, $b c r$-abl, est transcrit en un ARNm chimérique lui-même traduit en une protéine hybride de $210 \mathrm{kDa}$ ayant une forte activité tyrosine kinase. Cette anomalie cytogénétique, véritable marqueur de la maladie, est présente dans les mitoses de la lignée granuleuse et monocytaire des érythroblastes, des mégacaryocytes, des lymphocytes B 


\section{RÉFÉRENCES}

1. Clarkson B. Chronic myelogenous leukemia : is aggressive treatment indicated ? $J$ Clin Ontol 1985; 3 : 135-6.

2. Sokal JE, Cox EB, Baccarani M, et al. Pronostic discrimination in "good risk " chronic granulocytic leukemia. Blood 1984 ; 63 : 789-99.

3. Sokal JE, Baccarani M, Tura S, et al. Pronostic discrimination among younger patients with chronic granulocytic leukemia relevance to bone marrow transplantation. Blood 1985; 66 : 1352-7.

4. Talpaz M, McCredie KB, Mavligit GM, Gutterman JU. Leukocyte interferon induced myeloid cytoreduction in chronic myelogenous leukemia. Blood 1983; 82 . 689-92.

5. Talpaz M, Kantarjian HM, McCredie KB, Keating MJ, Trujillo J, Gutterman J. Clinical investigation of human alpha-interferon in chronic myelogenous leukemia. Blood 1987 ; 69 : 1280-8.

6. Alimena G, Morra E, Lazzarino M, et al. Recombinant alpha-2 interferon ( $\mathrm{r}$ IFN 2) in treatment of chronic myelogenous leukemia (CML). Blood 1988 ; 72 : 642-7.

7. Talpaz M, Kurzrock R, Kantarjian HM, Gutterman JV. Recent advances in the therapy of chronic myelogenous leukemia. In: De Vita VT, Hellman S, Rosenberg SA, eds. Important Advances in Oncology. Philadelphia: JB Lippincott, 1988: 297-321.

8. Niederle $\mathrm{N}$, Kloke O, May D Becher R, Osieka R, Schmidt CG. Treatment of chronic myelogenous leukemia with recombinant interferon alpha-2b. Invest New Drugs 1987 ; 5 (suppl) : 19-25.

9. Ozer H, Dear K, Testa J, et al. Prolonged administration of subcutaneous alphainterferon induces major clinical and complete cytogenetic remissions in untreated Philadelphia chromosome positive $\left(\mathrm{Ph}^{+}\right)$ chronic myelogenous leukemia. Proc $\mathrm{Am}$ Soc Clin Oncol 1990; $9: 783$. ct parfois des lymphocytes $\mathrm{T}$, dans de rares cas de transformation aiguë lymphoblastique $\mathrm{T}$ de $\mathrm{LMC}$.

L'évolution de la LMC se fait en deux temps: une première phase chronique ou myélocytaire, facilement contrôlée par les thérapeutiques usuelles, puis ensuite, parfois après une phase de transition appelée accélération, survient une transformation aiguë, souvent de type leucémie aiguë myéloblastique, terminale en trois à six mois. La survie médiane oscille entre trois et cinq ans, peu influencée par les monochimiothérapics classiques telles que l'hydroxyuréc ou le busulfan. Ces traitements permettent d'obtenir facilement et constamment une rémission hématologique, mais ils ne font pas disparaître le chromosome $\mathrm{Ph}$, celui-ci persistant dans $100 \%$ des mitoses médullaires. On a donc proposé des polychimiothérapies lourdes pour éliminer, si possible complètement, les cellules $\mathrm{Ph}+$ et espérer ainsi allonger la survic. Ces protocoles n'ont malheureuscment pas modifié significativement la survic (médiane entre 50 et 60 mois) ; surtout, ils sc sont avérés assez dangereux avec une morbidité non négligeable ct ont donc été abandonnés. Néanmoins, on a pu observer chez 20 à $53 \%$ des paticnts ainsi traités, une réduction des cellules $\mathrm{Ph}^{+}$en dessous de $35 \%$, avec même une rémission cytogénétique complète, certes transitoire, dans 10 à $33 \%$ des cas. Dans un de ces protocoles, la survie a semblé fonction de la réponse cytogénétique puisque les malades répondeurs (ceux ayant eu une rémission cytogénétique complète) ont présenté une médiane de survie à 90 mois contre 41 mois pour ceux ayant conservé $100 \%$ de mitoses $\mathrm{Ph}+[1]$.

Ultérieurement, l'allogreffe s'est imposée comme un traitement capable d'assurer une rémission cytogénétique complète et durable chez 50 à $70 \%$ des patients jeunes ayant un donneur HLA identique familial. Pour ces patients, on peut raisonnablement parler de guérison après trois ans de surveillance sans rechute. L'utilisation des interférons (IFN) est plus récente. Elle découle d'une observation simple: prescrits dans des essais thérapeutiques pour tumeurs solides, ils induisent une granulopénie. In vitro, ils inhibent la croissance des progéniteurs myéloïdes. La LMC (maladie où l'expansion myéloïde est très importante) semblait être une indication privilégiée pour les IFN. On a d'abord utilisé l'interféron leucocytaire, puis les interférons $\alpha-2 \mathrm{a}$ et $\alpha$-2b obtenus par génie génétique. Ces produits se sont avérés avoir une action bien supérieure aux chimiothérapies cytoréductrices classiques: ainsi, chez des patients mis en rémission hématologique, des analyses cytogénétiques médullaires régulières ont permis d'observer une baisse progressive du pourcentage des cellules $\mathrm{Ph}{ }^{+}$et, chez certains patients, une disparition complète de l'anomalie cytogénétique.

Néanmoins, ces résultats doivent être interprétés en tenant compte du risque relatif des patients. En effet, des études coopératives étudiant les paramètres préthérapeutiques de plus de 600 malades ont permis de montrer que l'âge, le sexe, la taille de la rate, le pourcentage des blastes sanguins, le chiffre des plaquettcs et l'hématocrite influençaient lc pronostic. Ces paramètres ont été inclus dans deux équations permettant, pour chaque patient, d'établir un indice de risque rclatif ou score pronostique de gravité. Trois groupes de patients ont pu être décrits : à risque faible (indice inférieur à 0,8 , survie 60 mois), à risque intermédiaire (indice entre 0,8 et 1,2 , survie 42 mois), à risque élevé (indice supérieur à 1,2 , survie 32 mois) [2, 3]. Les meilleurs résultats obtenus avec les interférons ont surtout été observés chez les patients à risque faible ou intermédiaire.

\section{Les interférons $\alpha$ : analyse des résultats}

Résultats des interférons utilisés seuls

Dans un premier travail, Talpaz avait montré, en 1983 [4], l'efficacité de l'IFN $\alpha$-leucocytaire partiellement purifié (IFN naturel) chez sept patients en phase chronique de LMC. Il notait déjà l'influence de la dose d'IFN, des échecs ayant été observés avec trois millions d'unités/jour alors qu'une réponse hématologique était obtenue avec neuf millions d'unités/jour. De plus, les 
Tableau 1

RÉSULTATS DES TRAITEMENTS DE LA LMC PAR IFN RECOMBINANT

\begin{tabular}{|c|c|c|c|c|c|c|c|}
\hline \multirow[t]{2}{*}{$\begin{array}{l}\text { Auteurs (réf.) } \\
\text { type IFN }\end{array}$} & \multirow[t]{2}{*}{$\begin{array}{c}\text { Nombre } \\
\text { de patients } \\
\text { évaluables }\end{array}$} & \multirow[t]{2}{*}{ Dose d'IFN } & \multicolumn{2}{|c|}{$\begin{array}{l}\% \text { de rémissions } \\
\text { hématologiques } \\
\text { complètes }\end{array}$} & \multicolumn{2}{|c|}{$\begin{array}{l}\text { \% de réponses } \\
\text { cytogénétiques }\end{array}$} & \multirow[t]{2}{*}{$\begin{array}{l}\text { Rémissions } \\
\text { cytogénétiques } \\
\text { complètes }\end{array}$} \\
\hline & & & $\begin{array}{c}\text { Diagnostic } \\
<1 \text { an }\end{array}$ & $\begin{array}{c}\text { Diagnostic } \\
>1 \text { an }\end{array}$ & $\begin{array}{c}\text { Diagnostic } \\
<1 \text { an }\end{array}$ & $\begin{array}{c}\text { Diagnostic } \\
>1 \text { an }\end{array}$ & \\
\hline $\begin{array}{l}\text { Talpaz [7] } \\
\alpha-2 a\end{array}$ & 45 & $5 \times 10^{6} \mathrm{Ul} / \mathrm{m}^{2} / \mathrm{J}$ & $69 \%$ & $25 \%$ & $42 \%$ & $19 \%$ & $8(17 \%)$ \\
\hline $\begin{array}{l}\text { Nielerle [8] } \\
\alpha-2 b\end{array}$ & 36 & $4 \times 10^{6} \mathrm{UI} / \mathrm{m}^{2} / \mathrm{J}$ & \multicolumn{2}{|c|}{$58 \%$} & \multicolumn{2}{|c|}{$22 \%$} & 0 \\
\hline \multirow{2}{*}{$\begin{array}{l}\text { Alimena [6] } \\
\alpha-2 b\end{array}$} & 63 & $2 \times 10^{6} \mathrm{Ulm}^{2} .3 / \mathrm{sem}$ & $38 \%$ & $15 \%$ & \multirow{2}{*}{\multicolumn{2}{|c|}{$70 \%$}} & \\
\hline & & $5 \times 10^{6} \mathrm{UI} / \mathrm{m}^{2} .3 / \mathrm{sem}$ & $50 \%$ & $37 \%$ & & & 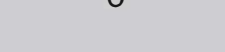 \\
\hline $\begin{array}{l}\text { Ozer [9] } \\
\alpha-2 b\end{array}$ & 112 & $5 \times 10^{6} \mathrm{UI} / \mathrm{m}^{2} / \mathrm{J}$ & \multicolumn{2}{|c|}{$84 \%$} & \multicolumn{2}{|c|}{$32 \%$} & $13 \%$ \\
\hline
\end{tabular}

analyses cytogénétiques régulières montraient la réapparition de 15 à $50 \%$ de cellules diploïdes normales. L'essai a été poursuivi sur 51 patients traités d'août 1981 à décembre 1984, et ayant un diagnostic récent de moins de six mois [5]. Talpaz a obtenu $71 \%$ de rémission hématologique (36 patients) avec un délai médian d'obtention de la rémission de 14 semaines (2 à 55). La rémission hématologique a été plus facilement obtenue chez les patients à faible risque $(80 \%)$ que chez ceux à risque élevé (64\%). Une réponse cytogénétique a été notée chez 20 des 36 patients ayant eu une rémission hématologique (soit $56 \%$ des patients ou $39 \%$ du total des malades entrés dans l'essai). La réponse a été minime (persistance de $35 \%$ à $95 \%$ de métaphases $\mathrm{Ph}^{+}$) chez 12 patients $(24 \%)$, partielle (persistance de 5 à $34 \%$ de métaphases $\mathrm{Ph}^{+}$) chez huit patients. La suppression du $\mathrm{Ph}$ a été lente à observer, l'amélioration survenant après une durée médiane de traitement de neuf mois (3 à 15). Cette amélioration cytogénétique a été aussi fonction des catégories de risque puisque observée chez $59 \%$ des patients à risque faible, alors qu'aucun des patients à risque élevé n'a eu d'amélioration cytogénétique. Celle-ci a été courte pour deux patients mais a duré au moins six mois chez 18 malades. Treize transformations aiguës (TA) ont été observées après un temps moyen de traitement de 11 mois : six lymphoblastiques, trois indifférenciées et quatre myéloïdes (9 TA étaient survenues chez des patients résistants ou en réponse partielle). Le suivi a été de 37 mois avec 40 patients en vic et une survie à trois ans de $76 \%$ (94\% pour ceux mis en rémission hématologique et $45 \%$ pour ceux en réponse particlle ou résistants). Le taux annuel de décès a été respectivement de $6 \%, 9 \%, 9 \%$ les trois premières années et donc inféricur à celui attendu chez des patients traités par chimiothérapie conventionnelle $(10,15,25 \%)$.

Les essais suivants ont été conduits avec des interférons recombinants $\alpha-2 \mathrm{a}$ ou $\alpha$-2b. Le Tableau I résume les résultats obtenus. La comparaison entre les études est cependant difficile pour plusieurs raisons. Les essais ont inclu des patients soit déjà traités plus ou moins longtemps par chimiothérapie conventionnelle, soit d'emblée dès le diagnostic, et cette hérérogénéité du recrutement doit être prise en considération pour l'appréciation des résultats. En effet, il persiste souvent au diagnostic des cellules $\mathrm{Ph}$ négatives, probablement non clonales mais qui vont disparaître après plusieurs mois d'évolution.
On doit donc s'attendre à une réponse au traitement différente selon que celui-ci est appliqué au diagnostic ou plus tard. La dose d'interféron a été variable d'un essai à l'autre: parfois on a donné une dose journalière, souvent $5 \times 10^{6} \mathrm{Ul} / \mathrm{m}^{2}$, mais aussi une dose, trois fois par semaine, variant de $2 \times 10^{6}$ à $5 \times 10^{6}$. L'appréciation de la réponse cytogénétique n'a pas été la même d'un travail à l'autre : ainsi dans l'étude d'Alimena [6], on parlait de réponse cytogénétique lorsque lc caryotype montrait une diminution d'au moins $5 \%$ du nombre de cellules $\mathrm{Ph}+$. Dans les études de Talpaz [7], la réponse cytogénétique a été décrite comme minime lorsqu'il y avait de 35 à $95 \%$ de cellules $\mathrm{Ph}+$ résiduelles, partielle avec de 5 à $34 \%$ de cellules $\mathrm{Ph}+$ et complète lorsqu'il n'y avait plus de cellules $\mathrm{Ph}+$. L'ensemble de ces travaux a cependant permis de réunir des informations précieuses. Ainsi la dose d'interféron joue un rôle puisque les meilleurs résultats ont été obtenus avec une dose journalière de $5 \times 10^{6} \mathrm{Ul} / \mathrm{m}^{2}$ administrée sans diminution pendant au moins les premiers mois du début du traitement. On a aussi noté que le maintien d'une certaine dose forte d'interféron semblait nécessaire pour induire et garder une cytopénie sur les globu- 


\section{RÉFÉRENCES}

10. Talpaz M, Kantarjian HM, Kurzrock R, Trujillo JM, Gutterman JV. Sustained complete cytogenetic responses among Philadelphia positive chronic myelogenous leukemia (CML $\mathrm{Ph}^{+}$) patients treated with alpha-interferon. Blood 1989 1 (suppl) : 74 : 9 a.

11. Bergsagel DE, Hass RH, Messner HA Interferon alpha-2b in the treatment of chronic granulocytic leukemia. Invest New Drugs 1987 ; suppl : 9-17.

12. Kantarjian HM, Talpaz M, Keating MJ, et al. Combined modality therapy with intensive chemotherapy (DOAP) followed by alpha-interferon (IFN-A) maintenance in chronic myelogenous leukemia (CML). Proc Am Soc Clin Oncol 1988; 29 : 210 .

13. Guilhot F, Dreyfus B, Desmarest MC, et al. Combined therapy interferon and chemotherapy in chronic myelogenous leukemia. Nouv Rev Fr Hematol 1989 ; 31 : 171-3.

14. Sokal JE, Gockerman JP, Gibner SH. Evidence for a selective antileukemic effect of cytosine arabinoside in chronic granulocytic leukemia. Leuk Res 1988; 12 : 453-8.

15. Guilhot F, Dreyfus B, Brizard A, Huret JL, Tanzer J. Cytogenetic remissions in chronic myelogenous leukemia using interferon alpha-2a and hydroxyurea with or without low-dose cytosine arabinoside. Leukemia Lymphoma 1991; 4 : 49-55.

16. Talpaz M, Kurzrock R, Kantarjian $H$, et al. Therapy of Philadelphia positive chronic myelogenous leukemia (CML Ph1) with recombinant alpha-2a interferon and recombinant gamma interferon. Blood 1988 ; 72 : 229a (abstr 828)

17. Yoffe M, Blick M, Kantarjian HM, Spitzer G, Gutterman J, Talpaz M. Molecular analysis of interferon induced suppression of Philadelphia chromosome in patients with chronic myeloid leukemia. Blood 1987 ; 69 : $961-3$.

18. Lee MS, Lemaistre A, Kantarjian $\mathrm{H}$, et al. Detection of two alternative brc-abl mRNA junction and minimal residual disease in Philadelphia chromosome positive chronic myelogenous leukemia by polymerase chain reaction. Blood 1989; 73 . 2165-70. les blancs et les plaquettes et celle-ci a semblé nécessaire pour obtenir une réponse cytogénétique. Le délai pour observer cette réponse a été variable d'une étude à l'autre, allant de six semaines pour celle de Niederle [8] jusqu'à six mois pour celle d'Ozer [9]. En moyenne, les rémissions hématologiques sont observées vers le troisième mois. La réponse cytogénétique est toujours plus tardive, survenant en moyenne vers le neuvième mois. Mais, dans la série d'Ozer, le délai médian pour la réponse cytogénétique maximale se situe vers le $18 \mathrm{e}$ mois et dans d'autres études, on a observé des rémissions cytogénétiques après deux ans de traitement. Qu'il s'agisse de rémissions hématologiques ou de réponses cytogénétiques, on note que celles-ci sont toujours plus importantes lorsque les patients sont traités rapidement après le diagnostic, dans les 12 mois au moins après celui-ci. Ainsi, dans l'étude de Talpaz, il y a deux fois plus de rémissions hématologiques pour les patients dont le diagnostic est inférieur à un an et presque toutes les études confirment que passé 12 mois, il est difficile lorsqu'un traitement par IFN est commencé, d'observer une réponse cytogénétique. Toutes les rémissions cytogénétiques complètes ont été observées chez des patients traités par interféron, rapidement après le diagnostic. L'influence du risque relatif est pratiquement constante avec (travail d'Alimena par exemple) $87,5 \%$ de rémissions hématologiques pour les patients à risque faible contre $14,4 \%$ pour ceux à risque élevé. Par ailleurs, dans cet essai italien, la réponse cytogénétique a été transitoire dans sept cas et a persisté six mois au plus dans 21 cas. Pour la majorité des malades, le taux le plus bas des cellules $\mathrm{Ph}+$ a été observé entre le $6^{\mathrm{e}}$ et le $12^{\mathrm{e}}$ mois de traitement. Enfin, sept crises blastiques ont été observées, 1 lymphoïde, 6 myéloïdes, survenant toutes chez des patients n'ayant pas répondu au traitement. Dans l'étude de Niederle, il n'y a pas eu de réponse cytogénétique complète mais 8 des 20 patients en rémission hématologique ont eu une réduction supérieure à $50 \%$ du taux des cellules $\mathrm{Ph}+$. Enfin, Talpaz a réactualisé récemment ses résultats
[10] en étudiant le devenir des 96 patients entrés dans ses deux essais thérapeutiques entre 1981 et 1988. Soixante-treize pour cent des malades ont été mis en rémission hématologique, 18 ont eu une rémission cytogénétique complète, 11 d'entre eux traités par l'IFN recombinant. Sur ces 18 patients, 11 ont eu une rémission cytogénétique d'une durée médiane de 30 mois (de plus de 6 mois à plus de 45 mois).

En résumé, il ressort de ces essais, la plupart non "randomisés ", que les interférons recombinants permettent d'obtenir des rémissions hématologiques et surtout des réponses cytogénétiques, ce dernier effet étant original car non obtenu avec les chimiothérapies classiques. Cette réponse cytogénétique s'observe chez les malades à l'indice de risque relatif faible ou intermédiaire, rapidement traités après le diagnostic et recevant une dose suffisante d'interféron pour induire et maintenir un état de pancytopénie. L'effet sur la survie a été peu ou pas étudié : dans l'étude d'Ozer, la survie à 24 mois est de $87,5 \%$ et le pourcentage de décès est inférieur à cclui qui aurait été observé si les patients avaient été traités par chimiothérapie conventionnelle $(14 \%)$.

Association interféron $\alpha$ et interféron $\gamma$ ou chimiothérapie

Pour améliorer les résultats obtenus avec l'IFN seul, on a testé la faisabilité et l'efficacité d'une association IFN et chimiothérapie. Plusieurs approches sont possibles : (a) utiliser un traitement initial chimiothérapique pour obtenir une rémission hématologique (protocoles polychimiothérapiques lourds, avec autogreffe de moelle osseuse, ou bien monochimiothérapie classique, busulfan ou hydroxyurée) et maintenir cette rémission hématologique par un traitement continu d'IFN ; (b) commencer simultanément l'IFN et la chimiothérapie dès le diagnostic ou dès l'entrée dans l'essai.

Bergsagel [11] a montré que l'IFN $\alpha-2 b$ à la dose de $2 \times 10^{6} \mathrm{Ul} / \mathrm{m}^{2}$, donné trois fois par semaine, pouvait maintenir une rémission hématologique induite par une chimiothérapie conventionnelle. Son étude reposait sur deux observations : (a) au cours 
de la LMC, on obscrve unc tendance à l'augmentation de l'index mitotiquc et unc perte de la capacité des cellules lcucémiques à sc différencier en cellulcs fonctionnelles; (b) l'IFN peut réduire la capacité d'autorenouvellement des ccllules myéloblastiques et myélomateuses. Ainsi, sept patients ont reçu du busulfan pour induire unc rémission hématologique puis, après arrêt de la chimiothérapic, lc temps de doublement leucocytaire et la durée de la rémission ont été déterminés durant la rechute hématologique. Après une deuxième administration de busulfan pour obtenir unc deuxième rémission hématologique, un traitement par IFN a permis, chez cinq paticnts, d'obscrver un ralentissement du temps de doublement leucocytairc avec prolongation de la durée de la rémission. Dans cettc étude, aucune réponse cytogénétique n'a été obscrvée.

Avec une autre approche, Kantarjian [12] a traité 32 paticnts par chimiothérapie lourde associant daunorubicine, vincristine, cytarabine, prednisone (DOAP). Après trois cycles de chimiothérapic lourde, il commençait un traitement d'entreticn par IFN $\alpha-2 b$ à la dose de 3 à $9 \times 10^{\text {i }} \mathrm{Ul} /$ jour. La chimiothérapie d'induction a permis d'obtenir une réduction des cellules $\mathrm{Ph}+$ à $0 \%$ chez 14 malades (44\%), de $5 \%$ à $34 \%$ chez sept patients supplémentaires $(22 \%)$ et de 35 à $95 \%$ chcz sept autrcs (22\%). Six patients ont eu unc suppression pcrsistante des métaphases $\mathrm{Ph}+$ sous traitement de maintenance par IFN pendant une duréc de suivi moyen de 25 mois. Après un suivi de 32 mois, 27 patients sont en vic, cinq sont décédés, deux en phase chronique, trois en crises blastiques. La survie à trois ans est de $84 \%$. Dans cette étude, la comparaison des courbes de survie montre un effet favorable net de l'association IFN/DOAP par rapport à l'utilisation d'IFN seul ou aux chimiothérapies lourdes ou à l'hydroxyuréc. Par aillcurs, la survie est différentc selon qu'il y a cu ou non une réduction de ccllules $\mathrm{Ph}+$ : la survie projctée à 48 mois cst de $100 \%$ pour les paticnts ayant cu unc réduction de cellules $\mathrm{Ph}+$ contrc $60 \%$ pour les patients sans réponse cytogénétique. Nous avons, à partir d'octobre 1986, commencé unc phase pilote combinant $\mathrm{cn}$ traitement d'induction, l'hydroxyurée (50 mg/ $\mathrm{kg} / \mathrm{jour}$ per os) et IFN $\alpha$-2a $(5 \times$ $10^{6} \mathrm{Ul} / \mathrm{m}^{2} /$ jour) [13]. Le traitement d'entreticn comportait de l'IFN à dose réduitc pour certains paticnts (de 3 à $5 \times 10^{6}$ Ul/jour, 5 jours sur 7) pour maintenir des chiffres de globules blancs proches de 3000 et ccux de plaquettes de 100 000. La cytosinc-arabinosinc (Ara-C) connuc pour avoir une action sélective sur les ccllulcs $\mathrm{Ph}^{+}$[14] a été rajoutéc cn cas de résistance cytogénétique à la dosc de 10 à $40 \mathrm{mg} / j$ jour, de 10 à 15 jours/mois. Sur 24 paticnts inclus dans cet essai, 12 avaicnt déjà été traités par chimiothérapie classique (hydroxyuréc ou busulfan) pendant unc période médianc de 31 mois (de 12 à 48 mois), 12 autres ont cu l'association chimiothérapie-IFN dès le diagnostic. Unc rémission hématologique a été obtenuc dans $75 \%$ des cas après un délai moyen de traitement de cinq semaincs. Une amélioration cytogénétiquc a été obscrvéc chez $54 \%$ des patients, en moyenne après six mois de traitement (de 3 à 9 mois). Sur les 24 paticnts, ncuf ont cu au moins une fois $0 \%$ de cellules $\mathrm{Ph}+$ (soit $37 \%$ ), six d'entre cux ont une réponse cytogénétique durablc avec unc médianc de duréc de réponse de 12 mois (de plus de 6 mois à plus de 30 mois). Parmi les dix paticnts ayant reçu l'association IFN-Ara-C, cinq ont cu une réponse cytogénétique complète dont trois durables, l'amélioration cytogénétique ayant été immédiate après l'introduction de l'Ara-C [15]. La survic de cette petite série de patients est de $88 \%$ à trois ans et demi. On pcut enfin signaler des essais d'association

Tableau II

RÉSULTATS DE LA PCR APRĖS TRAITEMENT PAR IFN

\begin{tabular}{|c|c|c|c|c|}
\hline \multicolumn{5}{|c|}{ Auteurs (réf.) } \\
\hline & Lee [18] & Opalka [19] & Bartram [20] & Martiat [21] \\
\hline $\begin{array}{l}\text { Nombre } \\
\text { de patients }\end{array}$ & 8 & 1 & 7 & 3 \\
\hline Type d'IFN & $\alpha-2 a$ & $\alpha-2 b$ & $\alpha-2 b$ & $\alpha$ \\
\hline Durée RCC & 6 mois à 3 ans & 13 mois & & \\
\hline Type PCR & 46 à 60 cycles & Techniques non décrites & Techniques non décrites & 2 PCR de 30 cycles chacune \\
\hline Résultats & $\begin{array}{l}8 \text { positifs } \\
6 \text { b2 a2 } \\
2 \text { b3 a } 2\end{array}$ & négatifs après 15 mois & $\begin{array}{c}5 \text { positifs } \\
2 \text { négatifs, } \\
\text { dont le cas d'Opalka }\end{array}$ & $\begin{array}{c}1 \mathrm{RCC} \text { à } 12 \text { mois } \mathrm{PCR}+\mathrm{b} 2 \text { a2 } \\
\text { puis négatif à } 24 \text { mois } \\
1 \mathrm{RCC} \text { à } 20 \text { mois PCR }{ }^{+} \\
\text {b3 a } 2 \\
1 \text { RCC à } 6 \text { mois PCR }{ }^{+} \\
\text {b3 a } 2\end{array}$ \\
\hline
\end{tabular}

RCC : rémission cytogénétique complète; b 2 a 2 : transcrit chimérique court exon b 2 de bcr + exon a 2 d'abl ; b 3 a 2 : transcrit chimérique long exon b3 de bcr + exon a2 d'abl. 
d'interférons : IFN $\alpha$ et $\gamma$ par exemple. Ce dernier active les cellules $\mathrm{T}$ et se fixe sur un récepteur différent. Une synergie a pu être espérée. En fait, les résultats ont été décevants et l'IFN $\gamma$, abandonné [16].

\section{RÉFÉRENCES}

19. Opalka B, Kloke O, Bartram CR, $e$ al. Elimination by interferon-alpha of malignant clone in chronic myeloid leukacmia. Lancet $1989 ; 1$ : 1334

20. Bartram CR, Yokota S, Hansen-Nagge TE, Janssen JWG. Detection of minimal residual leukemia by polymerase chain reaction. Bone Marrow Transplant 1990; 6 (suppl 1) : 4-8

21. Martiat P, Maisin D, Philippe M, et al. Detection of residual bcr/abl transcrits in chronic myeloid leukemia patients in complete remission using the polymerase chain reaction and nested primers. $\mathrm{Br} J \mathrm{Hae}$ matol $1990 ; 75$ : 355-8.

22. Talpaz M, Spitzer G, Hittelman W, Kantarjian H, Gutterman J. Changes in granulocyte-monocyte colony-forming cells among leukocyte-interferon-treated chronic myelogenous leukemia patients. Exp Hematol $1986 ; 14$ : 668-71.

23. Galvani DW, Cawley C. Mechanism of action of alpha-interferon in chronic myelogenous leukaemia : evidence for preferential inhibition of cate progenitors. $\mathrm{Br} J \mathrm{Hae}^{-}$ matol 1989 ; 73 : 475-9.

24. Rosenblum MG, Maxwelle BL, Talpaz M, Kelleher PJ, McCredie KB, Gutterman JU. In vivo sensitivity and resistance of chronic myelogenous leukemia cells to alpha-interferon. Correlation with receptor binding and induction of 2'5'-oligo adenylate synthetase. Cancer Res $1986 ; 46$ : 4848-52.

25. Demel WCP, Hoffbrand AV, Giles FJ, Goldstone AH, Mehta AB, Ganeshaguru K Alpha-interferon therapy for hameatological malignancies: correlation between in vivo induction of the 2',5' oligo adenylate system and clinical response. $\mathrm{Br} J$ Haematol 1990 ; $74: 452-6$.

26. Galvani DW, Owens W, Netherselle ABW, Cawley JL. The beneficial effects of alpha IFN in CGL are probably not mediated by NK cells. Br J Haematol $1989 ; 71: 233-7$

27. Daley GQ, Van Etten RA, Baltimore D. Induction of chronic myelogenous leukemia in mice by the P210 bcr/abl gene of the Philadelphia chromosome. Science 1990 ; 247 : 824-30

28. Singer JM, Arlin Z, Najfeld V, et al. Restauration of monoclonal hematopoiesis in chronic myelogenous leukemia (CML) following a chemotherapy-induced loss of the

\section{Résultats moléculaires du traite- ment par IFN}

L'analyse cytogénétique porte sur les cellules en mitose, alors que l'étude moléculaire apporte des informations sur l'ensemble des cellules, en division comme au repos. Deux types d'étude de la maladie résiduelle au niveau moléculaire sont possibles. Une première approche peut consister à rechercher un réarrangement par la technique de Southern. Ainsi deux patients traités par IFN, et en rémission cytogénétique complète depuis six mois, n'avaient plus de réarrangement [17]. Des expériences de dilution de l'ADN ont montré que la technique avait une sensibilité de l'ordre de $5 \%$. La recherche d'une maladie résiduelle par amplification génique par la réaction de la polymérase en chaîne (PCR) est plus intéressante. Cette technique, beaucoup plus sensible, permet de détecter une cellule maligne sur $10^{6}$ voire $10^{7}$. Le Tableau II, p. 457, résume les données actuellement disponibles à ce sujet. Les huit patients de Lee étaient positifs, ainsi que deux des patients de Martiat. L'un des deux patients de Bartram a rapidement été de nouveau positif et a présenté ensuite une rechute hématologique.

\section{Mécanismes d'action}

Il n'y a pas actuellement d'explications claires concernant le mécanisme d'action des interférons $\alpha$ dans la LMC. Un certain nombre de travaux ont été consacrés à l'étude des progéniteurs granulo-monocytaires : presque toutes les études ont démontré une action inhibitrice de la croissance des progéniteurs dans des systèmes de culture en milieu semisolide. Les cellules souches de la lignée granulo-monocytaire (GM$\mathrm{CFC)}$ diminuent progressivement après traitement par IFN et ceci est corrélée à la diminution de la cellularité médullaire. L'effet inhibiteur de l'IFN semble différent de ce qui est observé chez des patients traités pour tumeur solide et pourrait être lié à une action inhibitrice directe de l'IFN sur la capacité d'autorenouvellement des cellules progénitrices de la LMC [22]. L'inhibition de l'IFN pourrait porter sur des progéniteurs déjà engagés dans une certaine différenciation plutôt que sur des progéniteurs très indifférenciés [23]. Il semblerait, par ailleurs, qu'il $\mathrm{y}$ ait autant de récepteurs pour les IFN $\alpha$ sur les cellules $\mathrm{Ph}+$ que sur les cellules $\mathrm{Ph}^{-}$. Après fixation de l'IFN sur un récepteur membranaire, il y a induction de la 2',5' oligo adénylate synthétase (2-5A) et ensuite activation d'une ribonucléase. Certains auteurs [24] ont suggéré que l'augmentation de l'activité 2-5A pouvait être corrélée à la réponse thérapeutique. Ce résultat ne fait cependant pas l'unanimité et il semblerait qu'il faille distinguer la réponse in vivo de la réponse in vitro à l'induction de la $2-5 \mathrm{~A}$ : seule la première semblant corrélée à la réponse à l'IFN $\alpha$ [25]. Il n'y a pas $\mathrm{d}$ 'argument pour une action indirecte des IFN $\alpha$ dans la LMC. Bien que l'activité natural-killer $(\mathrm{NK})^{*}$ soit diminuée dans la LMC, l'administration des IFN $\alpha$ ne restaure pas cette activité. De plus, dans des expériences in vitro, on note que l'activité des IFN $\alpha$ persiste même si la moelle a été déplétée au préalable en cellules NK [26]. De la même manière, l'activité cytotoxique des lymphocytes $\mathrm{T}$ est normale dans la LMC et n'augmente pas après administration d'IFN. Enfin, l'activité des IFN alpha ne passe pas par la stimulation de la production du $\mathrm{TNF} \alpha$ : le blocage de son activité par des anticorps anti-TNF n'abolit pas l'action des IFN $\alpha$ [26].

Quel que soit leur mécanisme d'action, l'originalité thérapeutique des IFN $\alpha$ doit être soulignée: ils induisent, seuls ou associés à une chimiothérapie, une rémission cytogénétique complète chez 15 à $35 \%$ des patients et une bonne réponse cytogénétique $(\mathrm{Ph}+<35 \%)$ chez 10 à $20 \%$ de patients supplémentaires. Les données historiques dont on dispose (suivi des patients traités par chimiothérapie lourde) suggèrent qu'un gain appréciable sur la survie

* Voir Lexique Immunologie, suppl. au $n^{\circ} 1$, vol. 5 , p. 18. 
est à espérer pour ceux des patients ayant présenté une réponse cytogénétique. Mais les cellules $\mathrm{Ph}+$ représentent-elles le clone malin et leur élimination restaure-t-elle une hématopoï̀se non clonale ? Plusieurs données fondamentales permettent de penser que l'éradication des cellules $\mathrm{Ph}+$ est bien une étape indispensable pour la guérison et qu'une hématopoïèse non clonale est retrouvée. Ainsi, récemment, a-t-on pu démontrer le rôle pathogène primordial du gène hybride $b c r$-abl : des souris infectées par une construction rétro-virale contenant le gène $b c r$-abl ont effectivement présenté un syndrome hématologique du type de celui de la LMC [27]. Singer et al. ont rapporté le cas d'une femme hétérozygote pour les variants de la G6PD et dont l'hématopoïèse était entièrement clonale au diagnostic avec synthèse uniquement du variant A. Après chimiothérapie intensive, $75 \%$ des cellules médullaires étaient redevenues $\mathrm{Ph}$ négatives et les deux variants $\mathrm{A}$ et $B$ étaient retrouvés dans les globules rouges, les globules blancs et les plaquettes [28].

\section{Conclusion}

Bien qu'il reste de nombreuses incertitudes, il semblerait que les IFN $\alpha$ puissent représenter, dans le traitement de la LMC, une alternative raisonnable lorsqu'une allogreffe de moelle osseuse n'est pas possible. Ils permettent d'obtenir une rémission hématologique et, dans un nombre non négligeable de cas, une rémission cytogénétique complète. Ces résultats cytogénétiques peuvent être encore améliorés par l'adjonction de cures de chimiothérapie comme la cytosinearabinoside à petite dose. Bien que l'effet réel de cette nouvelle stratégie thérapeutique sur la survie reste indéterminé, on peut espérer que celle-ci sera améliorée par l'obtention d'une rémission cytogénétique complète. Cela est suggéré par les résultats des protocoles de chimiothérapie lourde et par le rôle pathogène fondamental du gène hybride $b c r$-abl

Ce travail a été réalisé grâce à une subvention de l'ARC.

$m / s n^{\circ} 5$, vol. 7 , mai 91

\section{Summary}

Treatment of chronic myelogenous leukemia by $\alpha$-interferon

Life expectancy of patients treated by conventional chemotherapy for chronic myelogenous leukemia (CML) has not changed during the past 30 years and the only curative therapy available up to now has been bone marrow transplantation. It is now demonstrated that human natural and recombinant interferons (IFN) suppress growth and differentiation of normal and CML progenitor cells in vitro and induce granulocytopenia in vivo. Alpha IFN has been shown to induce complete hematological remission in 60 to $80 \%$ of cases of CML patients. Its efficiency is dependent on the dose of IFN, the risk category of the patients and the duration of the disease before treatment. In some patients (15 to $35 \%$ ) complete cytogenetic response has been reported. Patients who are not candidates for transplant may be eligible for trials involving interferons. Although the effect of IFN alpha on survival cannot yet be ascertained, the finding that this agent suppresses the $\mathrm{Ph}$ positive clone provides an extremely attractive basis for its use in CML. 